\title{
Group Forecasting Accuracy in Hotels
}

\author{
Sheryl E. Kimes \\ Cornell University
}

Published: The Journal of the Operational Research Society (1999), 50(11), 1104-1110

Send correspondence to Sheryl E. Kimes (email: sek6@ cornell.edu) School of Hotel Administration, Cornell University, Ithaca, NY 14853-6902.

This paper was developed with the assistance of Y. Lee and E. Roekaert.

Keywords: yield management; hotels; forecasting; groups 


\begin{abstract}
Yield management helps hotels more profitably manage the capacity of their rooms. Hotels tend to have two types of business: transient and group. Yield management research and systems have been designed for transient business in which the group forecast is taken as a given. In this research, forecast data from approximately 90 hotels of a large North American hotel chain were used to determine the accuracy of group forecasts and to identify factors associated with accurate forecasts. Forecasts showed a positive bias and had a mean absolute percentage error (MAPE) of $40 \%$ at two months before arrival; $30 \%$ at one month before arrival; and 10-15\% on the day of arrival. Larger hotels, hotels with a higher dependence on group business, and hotels that updated their forecasts frequently during the month before arrival had more accurate forecasts.
\end{abstract}




\section{Introduction}

Yield management, a method for profitably managing capacity, has gained widespread acceptance in the hotel industry. Yield management is a method which helps hotels sell the right room to the right type of customer, at the right time and for the right price. ${ }^{1}$ Yield management guides the decision of how to allocate rooms to available demand in such a way as to maximize profit or revenue. The problem then becomes one of determining how much to sell at what price and to which market sector. The application of yield management has been most effective when applied to industries with relatively fixed capacity, predictable demand, perishable inventory, an appropriate cost and pricing structure and time-variable demand. ${ }^{1,2}$ Yield management providers and users commonly tout revenue improvements of 2-7\%.,4

Yield management systems rely on demand forecasts. Some yield management systems use forecasts of daily rooms occupied, while more sophisticated systems base decisions on the daily number of arrivals (by length of stay and room rate). Night room methods include the threshold curve, ${ }^{1}$ availability control ${ }^{5}$ and expected marginal seat revenue ${ }^{6}$ approaches. Arrival systems include mathematical programming ${ }^{7}$ and bid price ${ }^{5,8}$ approaches.

Hotel yield management research has focused on room sales to individuals (transient business), and has used the group forecast as given. ${ }^{9}$ Yield management systems rely on accurate transient and group forecasts as inputs. Transient forecasts are used to determine the rate and availability restrictions, while group forecasts are used to calculate the number of rooms available to sell to transient guests. If the group forecast is inaccurate, the number of rooms available for sale will be inaccurate, and the recommendations of the yield management system may lead to poor decisions. The intent of this research was to examine the extent of group forecast error and to identify factors associated with accurate group forecasts.

Two questions emerge: (1) how accurate are group forecasts? and (2) what factors impact the accuracy of group forecasts? In order to study these questions, group forecasting data were obtained from a major hotel chain for approximately 90 different hotel properties. The data were used to calculate group forecasting errors and to identify factors associated with group forecasting error.

\section{Hotel group business}

Groups constitute a substantial amount of hotel business. No systematic published research on the composition of hotel business has been conducted. Demand composition data were collected from two upscale hotel chains and analyzed to determine the average percentage of rooms sold to groups. Approximately $38 \%$ of all rooms were sold to groups. Resort and downtown hotels had a slightly higher reliance on group business (45\% and $42 \%$ respectively), while airport and suburban hotels had a slightly lower reliance on group business (32\% and 34\% respectively).

Group business is not homogeneous and consists of groups from various market segments (namely, national associations, corporate meetings, religious groups, and local social events) and of varying sizes. Groups typically book a block of rooms 6 months to $2 \mathrm{yr}$ in advance, and group members make reservations directly with the hotel. The group room block is held until a contracted release date (usually one month before scheduled arrival).

Hotels usually have multiple groups of varying size for each day. The sales manager responsible for each group provides the director of sales with a daily forecast of rooms sold 
based on reservation pick-up, past performance and communications with the group meeting planner. The director of sales then adjusts and compiles these forecasts and presents them to the reservations manager. The reservations manager adjusts the forecast and enters it into the yield management system. More sophisticated group forecasting methods have been suggested ${ }^{10}$ but for the most part, group forecasting methods are qualitative in nature.

Opportunities for bias exist throughout the system. ${ }^{11}$ Sales managers often receive bonuses based on the number of rooms booked rather than the number of rooms sold. ${ }^{12}$ This may lead to overly optimistic forecasts, especially when combined with the high turnover of group sales managers, by the need to achieve budgeted sales and the long lead time associated with groups. The director of sales may also be affected by the bonus system and by achieving budgeted sales quotas.

\section{Literature review}

Forecasting for transient and group business serves different purposes. Transient forecasts predict future demand, while group forecasts predict demand materialisation. ${ }^{13,14}$ Groups request room blocks far in advance and are not required to use all of the rooms. ${ }^{15}$ Because of this, sales managers must adjust the size of the room block to reflect the number of rooms which will actually be sold.

Both transient and group forecasts are based on historical and booking pace information, but the type of data used varies. Transient forecasting relies on historical demand, booking profiles, and managerial knowledge of the market, ${ }^{16}$ while group forecasting is dependent upon historical utilization, group booking profiles and sales estimates of the group size.

Research on group forecasting is minimal. Guidelines for developing group forecasts in the hotel industry have been suggested, ${ }^{10,15,17}$ but no formal research has been published. Limited research on group forecasting has been conducted in the airline industry ${ }^{13}$ and the cruise line industry. ${ }^{14}$

The primary emphasis of Svrcek's ${ }^{13}$ research was on the allocation of airline seats to group demand, but he also addressed the issue of group forecasting. He used discrete transform analysis to combine the number of group requests, the size of each request and the utilization ratios into a single probability distribution which could then be used to forecast group demand.

Fisher and Mongalo ${ }^{14}$ described the development of yield management at Royal Caribbean Cruises. Integral to the yield management system was a retention forecasting system which was designed to predict the number of booked passengers who would actually sail. The retention forecasting system used a 2 y moving average of historical data to quantify factors affecting retention. Factors considered included ship, group type, payment status, agent type, itinerary, time of year and time to departure.

Lee ${ }^{16}$ studied the impact of forecast accuracy on revenue production in the airline industry by varying the mean and standard deviation of the forecast for four different simulated demand scenarios (low, medium, high and very high). Variations in the standard deviation had little impact on expected revenues, but variations in the mean produced substantial changes in expected revenues in high and very high demand situations. Over-forecasting errors caused a larger decline in revenue than under-forecasting errors. When demand was over-forecast, lower fares were overly restricted, while when demand was under-forecast, fares were not sufficiently restricted. On high and very high demand flights, a 10\% improvement in forecast accuracy increased revenue by $0.5 \%-3.0 \%{ }^{16}$ 


\section{Description of the study}

Group forecasting data were obtained for approximately 90 hotels of a major North American hotel chain. Four months of daily data consisting of group forecasts and updates for the two months prior to each date were obtained. In addition, data on transient forecasts and final group and transient rooms sold were collected.

The company did not track forecast information for each day before arrival, but instead updated the number of reservations on hand on selected days, (reading days). Reading days are commonly used in the hotel and airline industries because of the reduced amount of data required. The subject hotel chain updated their forecast data daily during the two weeks before arrival, but only updated weekly during the 2-8 weeks before arrival. In total, updated forecast data were available at 22 different reading days $(0-15,22,29,36,43,50$, and $57 \mathrm{~d}$ before arrival (DBA)).

The hotels ranged in size from 200 rooms to over 1500 rooms and comprised a variety of hotel types including resort, downtown, suburban, and airport properties. The hotel chain used a yield management system and reservations managers at each hotel were responsible for updating group and transient forecasts for each arrival date for the next two to three months.

\section{Impact and measurement of group forecast error}

Inaccurate group forecasts have more impact during high occupancy times. If group forecasts are too high, transient business (which usually pays a higher room rate) may be unnecessarily displaced, and the hotel may find itself with empty rooms which could have been sold. Conversely, if the group forecast is too low, excess transient reservations may be accepted and the hotel may end up overselling its capacity or selling its transient rooms at too low of a rate. When occupancies are low, inaccurate forecasts have little impact other than causing incorrect staffing and purchasing decisions.

Forecast error is the difference between the group forecast and the number of group

rooms actually sold. While many forecast error measurements are available, ${ }^{18}$ hotel managers are usually interested in knowing the percentage of the error, the size of the error (the number of rooms sold), and the direction of the error (too high or too low). Because of this, the Mean Absolute Percentage Error (MAPE), the Mean Absolute Deviation (MAD), and the Mean Forecast Error (MFE) were calculated to determine the forecast accuracy for each hotel for each reading day.

\section{Factors affecting group forecast error}

Group forecast accuracy may be affected by the (1) time before arrival; (2) type of hotel; (3) size of the hotel (also serving as a proxy for the size of the sales office); (4) occupancy of the hotel; (5) dependence on group business; (6) frequency of forecast updates; (7) timing of forecast updates; and (8) number of groups in the hotel. Data on each of the first seven factors were available, but information on the number of groups at each hotel for each day during the study period was unavailable. Since the hotels studied were all from the same chain, other factors such 
as global distribution system access and national sales forces were not included because all hotels had access to the same resources. Specific definitions of each factor were:

(1) Time before arrival: Days before arrival (DBA)

(2) Type of hotel: Resort, airport, suburban or downtown

(3) Size of hotel: About 200 to over 1500 rooms.

(4) Occupancy: Occupancy percentage

(5) Dependence on group business: Final number of group rooms sold divided by the total number of rooms sold.

(6) Frequency of forecast updates: Average number of forecast updates

(7) Timing of forecast updates: When forecasts were updated

\section{Results}

The MAD, MAPE and bias of group forecast error (MFE) were calculated for each hotel for each arrival date and for all associated reading days. The MAPE was calculated by dividing the MAD by the actual number of rooms sold to groups. All error terms (MAD, MAPE, MFE) were normally distributed. Following this calculation, the impact of each of the seven factors affecting group forecast error (specifically the MAPE) was analyzed. A bivariate analysis was performed, even though this problem may be multivariate in nature. An analysis of possible inter-correlations was performed, and in general, the factors studied were not highly correlated, but larger hotels had a higher percentage of group business $(r=0.491)$ as did downtown hotels $(r=0.389)$ (Table 1).

\section{Insert Table 1 Here}

\section{Time before arrival}

MAPE: As expected, group forecast accuracy improved as the day of arrival approached. In general, the MAPE averaged $40 \%$ at two months before arrival, dropped to about $30 \%$ at one month before arrival, and decreased to $10-15 \%$ on the day of arrival (Figure 1). As the day of arrival approached, the standard deviation of the MAPE decreased from approximately $22 \%$ at two months before arrival, to about $16 \%$ at one month before arrival, to around $11 \%$ on the day of arrival.

$M A D$ : The MAD decreased from approximately 70 rooms at two months before arrival to 40 rooms at one month before arrival to approximately 20 rooms on the day of arrival (Figure 2). The standard deviation of the MAD decreased from approximately 65 rooms at two months before arrival, to 50 rooms at one month before arrival, to approximately 15 rooms on the day of arrival.

$M F E$ : The Mean Forecast Error (MFE) for each hotel was calculated to determine forecast bias. Hotels tended to over-forecast, especially when near the day of arrival. The group forecasts were relatively unbiased at two months before arrival (Figure 3). 
Insert Figure 1 Here

\section{Type of hotel}

Hotels were classified as either resort, airport, suburban, or downtown hotels. The MAPE, MAD and MFE for each hotel type was calculated for each reading day. Error patterns were relatively similar, although resort hotels and airport hotels showed a higher MAPE for all days before arrival (Figure 4). ANOVA tests were conducted to see if forecast accuracy varied by type of hotel. In general, the type of hotel did not impact group forecast accuracy. $I$

\section{Size of the hotel}

Correlations between the number of rooms in a hotel and group forecast accuracy were calculated for each reading day. Larger hotels had better forecast accuracy $(r=-0.20$ to $-0.31, P<0.05$ ). In addition, hotels were classified as small (less than 400 rooms), medium (400-799 rooms) or large (800 rooms or more). ANOVA tests for each reading day showed a significant difference in forecast accuracy among different sized hotels for each reading day $(P<0.05)$.

\section{Insert Figure 2 Here}

\section{Hotel occupancy rate}

The daily occupancy rate for each hotel was calculated and correlated with the MAPE for each reading day. There was little relationship between occupancy rate and forecast accuracy $(r=-0.1$ to -0.2$)$. ANOVA tests showed the same result.

\section{Insert Figure 3 Here}

\section{Dependence on group business}

The reliance on group business was calculated for each hotel by dividing the number of group rooms sold by the total number of rooms sold. Correlations comparing the reliance on group business and group forecast accuracy were computed ( $r=-0.35$ to -0.47$)$. In addition, hotels were classified as primarily transient (less than $25 \%$ group), mixed (25-50\% group) or primarily group (more than 50\% group). ANOVA tests were conducted for each reading day to see if forecast accuracy varied by group business percentage. As reliance on group business increased, group forecast accuracy improved $(P<0.05)$. 
Insert Figure 4 Here

\section{Frequency of changes}

All hotels had access to updated information, but some chose to update their forecasts frequently, while others never updated their forecasts. The average number of forecast updates was calculated for each hotel and correlations between the number of updates and the group forecast accuracy for each day before arrival were calculated. There was a negative correlation between the number of updates and forecast accuracy during the week before arrival $(r=-0.4$ to -0.5). ANOVA tests were conducted to see if update frequency had an effect on forecast accuracy. Hotels were classified as no forecast updates, few forecast updates (1-5 updates), some forecast updates (6-10 updates), frequent forecast updates (11-15 updates), or constant forecast updates (more than is 15 updates). Frequency of update was associated with increased forecast accuracy from $0-11 \mathrm{~d}$ before arrival $(P<0.05)$.

\section{Timing of forecast updates}

Hotels updated their forecasts at different times before arrival, so the timing of each update was determined for each hotel. Updates were classified as either early (4-8 weeks before arrival), medium-term (2-4 weeks before arrival), late (1-2 weeks before arrival), or very late (less than 1 week before arrival). Correlations between the timing of the updates and the MAPE were computed to test the relationship between updates made at different reading days. For example, hotels that updated early did not necessarily make late updates. The number of early updates were correlated with the number of medium updates $(r=0.32)$, the number of late updates $(r=0.36)$, and the number of very late updates $(r=0.21)$. The number of mediumterm updates was highly correlated with late $(0.77)$ and very late $(0.59)$ updates, and the number of late updates was highly correlated with the number of very late updates $(0.84)$. This means that hotels that performed medium-term and late updates tended to also perform short-term and very-short term updates.

The correlation between the update timing and forecast accuracy was also calculated (Table 2). Early updates (those more than 4 weeks before arrival) showed no correlation with forecast accuracy, while the number of medium-term (2-4 weeks before arrival) updates was negatively correlated with the MAPE for $0-7 \mathrm{~d}$ before arrival $(r=-0.23$ to -0.31$)$. The number of late updates (1-2 weeks before arrival) and the number of very late updates (less than one week before arrival) were also negatively correlated with the MAPE for 0-7 $\mathrm{d}$ before arrival (late, -0.26 to -0.38 ; very late, -0.17 to -0.35 ). Early forecast updates had little impact on forecast accuracy, but frequent updates during the month before arrival were associated with reduced forecast error. 


\section{Summary and conclusions}

Hotels with lower group forecasting error tended to be larger, depend more on group business, make frequent updates, and update frequently during the two weeks before arrival. The type and occupancy of the hotel did not have a major impact on group forecasting error.

Although additional research is needed to fully understand the reasons for these relationships, several explanations may be appropriate. Larger hotels with a high dependence on group business may have more experienced staff dealing with groups. These experienced employees may have learned that frequent updates are key to the reduction of forecast errors. In addition, the aggregation of error from the increased number of groups at larger hotels may serve to balance out group forecast error. Multivariate analysis may help address some of these questions. Future research will address the issue of best practices of hotels with low group forecasting error.

The revenue impact of group forecast error is currently under study. Clearly, group forecast error has more effect on revenue generation when a hotel is experiencing high occupancy and possible transient displacement. In order to study transient displacement, the level of analysis must be changed from a monthly comparison of hotels to detailed daily analysis of individual hotels.

A clear understanding of group forecasting and group forecasting error is essential to the successful implementation of yield management techniques in hotels and other capacityconstrained services. Since yield management systems take the group forecast as a given, every effort should be taken to ensure that the group forecast is as accurate as possible. The results of this research indicate that frequent updates are associated with increased group forecast accuracy. 
Table 1. Correlation analysis.

\begin{tabular}{|c|c|c|c|c|c|c|c|c|}
\hline & $\begin{array}{c}\text { Size } \\
\text { (rooms) }\end{array}$ & $\begin{array}{c}\text { Convention } \\
\text { hotel }\end{array}$ & $\begin{array}{c}\text { Occupancy } \\
\%\end{array}$ & $\begin{array}{l}\% \text { of } \\
\text { group }\end{array}$ & $\begin{array}{c}\text { Downtown } \\
\text { hotel }\end{array}$ & $\begin{array}{c}\text { Airport } \\
\text { hotel }\end{array}$ & $\begin{array}{c}\text { Suburban } \\
\text { hotel }\end{array}$ & $\begin{array}{c}\text { No. of } \\
\text { updates }\end{array}$ \\
\hline Size (rooms) & 1.000 & & & & & & & \\
\hline Convention hotel & 0.022 & 1.000 & & & & & & \\
\hline Occupancy \% & 0.023 & -0.275 & 1.000 & & & & & \\
\hline$\%$ of group & 0.491 & 0.236 & 0.110 & 1.000 & & & & \\
\hline Downtown hotel & 0.208 & 0.261 & -0.060 & 0.389 & 1.000 & & & \\
\hline Airport hotel & 0.148 & 0.003 & -0.082 & -0.107 & -0.363 & 1.000 & & \\
\hline Suburban hotel & -0.311 & -0.271 & 0.115 & -0.330 & -0.792 & -0.281 & 1.000 & \\
\hline No. of updates & -0.142 & 0.050 & -0.088 & -0.198 & -0.303 & 0.270 & 0.136 & 1.000 \\
\hline
\end{tabular}


Table 2. Correlation between timing of updates and MAPE

\begin{tabular}{ccccc}
\hline DBA & $\begin{array}{c}\text { 4-8 weeks before } \\
\text { arrival }\end{array}$ & $\begin{array}{c}2-4 \text { weeks before } \\
\text { arrival }\end{array}$ & $\begin{array}{c}1-2 \text { weeks before } \\
\text { arrival }\end{array}$ & $\begin{array}{c}\text { Less than 1 week } \\
\text { before arrival }\end{array}$ \\
\hline 0 & -0.056 & -0.309 & -0.381 & -0.347 \\
7 & -0.091 & -0.232 & -0.270 & -0.166 \\
14 & -0.081 & -0.182 & -0.190 & -0.108 \\
29 & -0.140 & 0.046 & -0.117 & -0.028 \\
57 & -0.076 & 0.033 & 0.014 & 0.077 \\
\hline
\end{tabular}




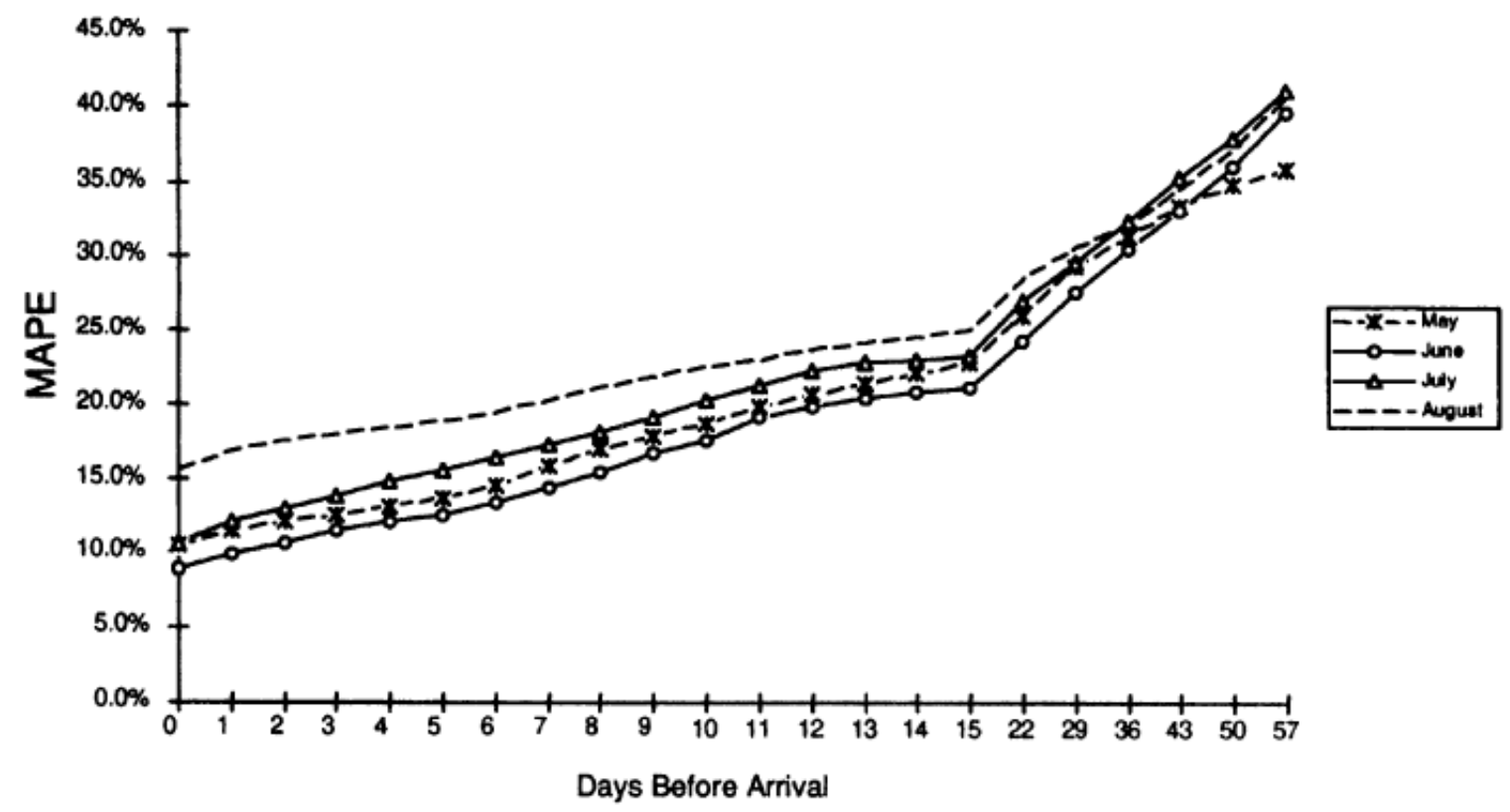

Figure 1. Mean absolute percentage error (MAPE) of group forecast. 


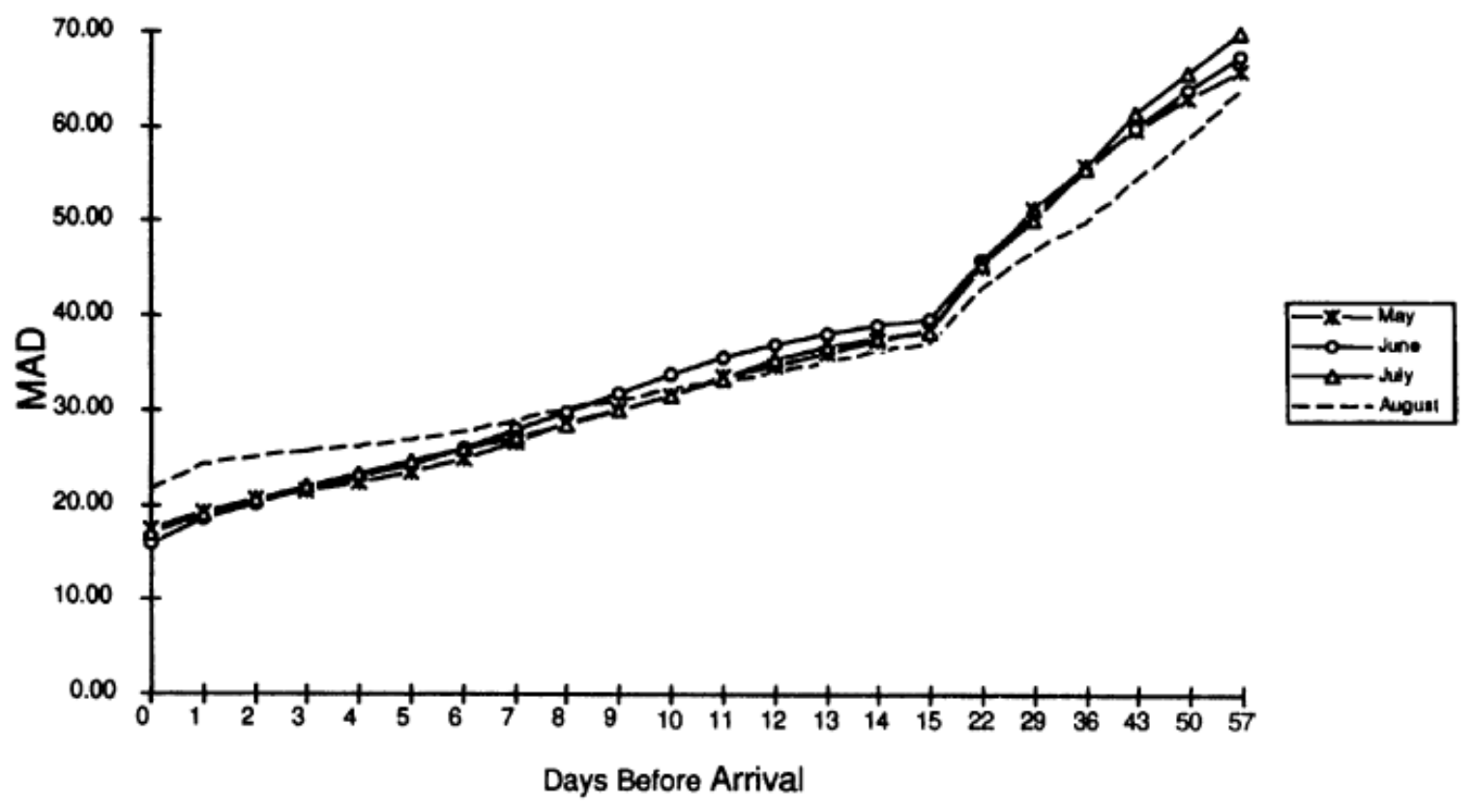

Figure 2. Mean absolute deviation (MAD) of group forecast. 


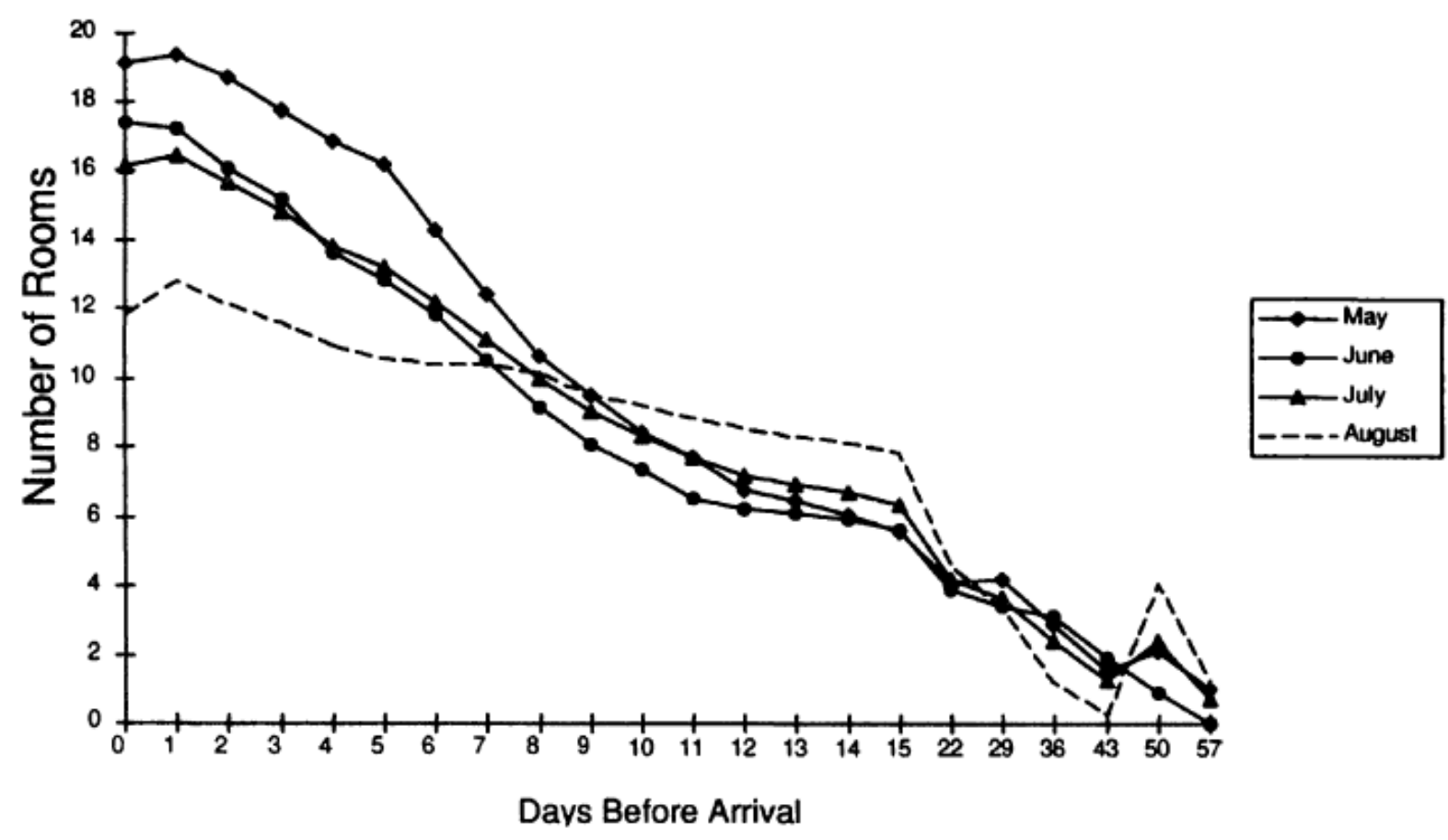

Figure 3. Mean forecast error (number of rooms). 


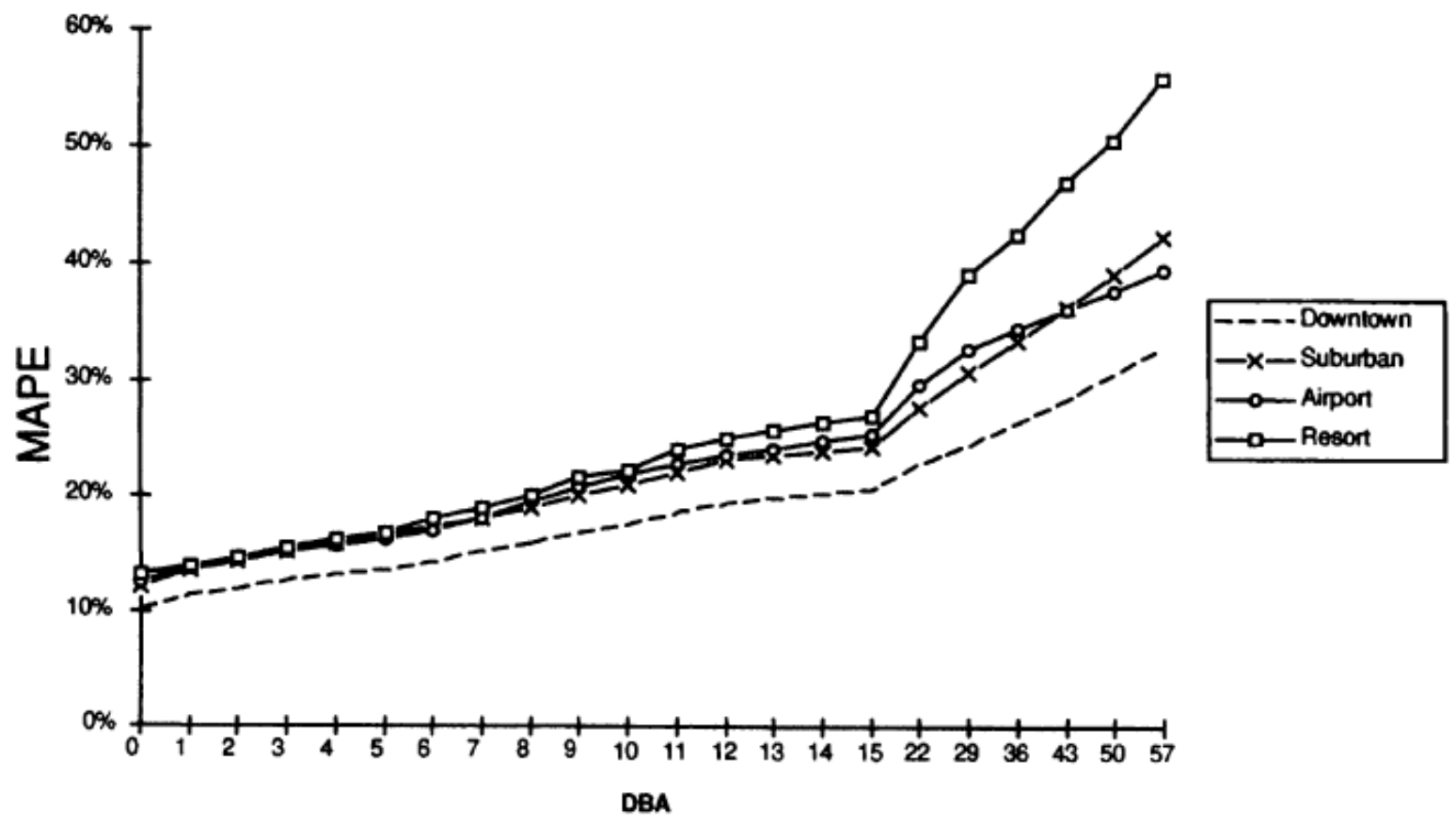

Figure 4. Group forecast MAPE by hotel type. 


\section{References}

$1 \quad$ Kimes SE (1989). Yield management: a tool for capacity constrained service firms. $J O p$ Mgmt 8 (4): 348-363.

2 Cross RG (1997). Revenue Management: Hard-Core Tactics for Market Domination. Broadway Books: New York.

3 Smith B, Leimkuhler JF and Darrow RM (1992). Yield management at American Airlines. Interfaces 22 (1): 8-31.

$4 \quad$ Hanks RD, Cross RG and Noland RP (1992). Discounting in the hotel industry: a new approach, Cornell Hotel and Restaurant Admin Q May: 4045.

5 Belobaba PP (1987). Air travel demand and airline seat inventory management. $\mathrm{PhD}$ Thesis, Massachusetts Institute of Technology.

6 Williamson EL (1992). Airline network seat control. PhD Thesis, Massachusetts Institute of Technology.

$7 \quad$ Vinod B (1995). Origin-and-destination yield management. In: Jenkins, D (ed). Handbook of Airline Economics. McGraw-Hill: New York, pp 649-658.

8 Kimes SE and Chase RB (1998). The strategic levers of yield management. J Service Mgmt 1: 158-168.

9 Weatherford LR and SE Bodily (1992). A taxonomy and research overview of perishable-asset revenue management: yield management, overbooking and pricing. Opns Res 40: 831-844.

10 Kimes SE (1999). Group forecasting methods for hotels. Res Forum of the Cornell Hotel and Restaurant Admin $Q$ (In press).

11 Mahmoud E, DeRoeck R, Brown R and Rice G (1992). Bridging the gap between theory and practice in forecasting. Int $J$ of Forecasting 8: 251-267.

12 Freitag H (1998). Establish sales incentives that work best for the property. Hotel and Motel Mgmt May 18.

13 Svrcek T (1991). Modeling airline group passenger demand for revenue optimization. Flight Transportation Laboratory Report R91-5. Massachusetts Institute of Technology

14 Fisher J and Mongalo M (1993). Integrating decision support. SAS Users Groups International Conference.: New York: SAS Group, 619-623.

15 Hoyle LH, Dorf DC and Jones TJA (1991). Managing Conventions and Group Business. The Educational Institute of the American Hotel and Motel Association: Washington, DC.

16 Lee A (1990). Airline reservations forecasting: probabilistic and statistical models of the booking process. Flight Transportation Laboratory Report R90-5. Massachusetts Institute of Technology

17 Vallen JJ and Vallen GK (F991). Check-in Check-out. William C. Brown Publishers: Dubuque, Iowa.

18 Armstrong JS and Collopy F (1992). Error measures for generalizing about forecasting methods: empirical comparisons. Int $J$ of Forecasting 8: 69-80. 